\title{
Limiar de lactato em exercício resistido em idosos
}

\section{Lactate threshold during resistance exercise in elderly}

\author{
N.F. Sousa, M.C. Souza, G.B. Pereira, D.R. Bertucci, R.F. Magosso, V. Baldissera, S.P. \\ Andrade
}

ARTIGO ORIGINAL | ORIGINAL ARTICLE

\begin{abstract}
A presença do limiar anaeróbio (LAn) em exercício resistido tem sido objeto de estudo nos últimos anos. O objetivo do estudo foi identificar e comparar o LAn em uma população idosa no leg press (LP) e supino reto (SR). Catorze homens idosos $(68.9 \pm 4.0$ anos, $170.5 \pm 0.05 \mathrm{~cm}$ e $76.1 \pm 7.8$ $\mathrm{kg}$ ) realizaram dois testes incrementais máximos baseados em porcentagens de uma repetição máxima (1RM) para a determinação do LAn no LP e SR. As variáveis lactacidemia, frequência cardíaca (FC) e percepção subjetiva de esforço (PSE) foram coletadas ao final de cada estágio e comparadas entre os exercícios na intensidade referente ao LAn. O nível de significância adotado foi $p \leq .05$. O LAn no LP ocorreu em $27.9 \pm 5.0 \%$ de $1 \mathrm{RM}$, estatisticamente superior ao SR, que foi identificado em $21.5 \pm 3.1 \%$ de 1RM. No entanto, a lactacidemia foi estatisticamente inferior no LP (1.36 $\pm 0.40 \mathrm{mmol.L}-1) \mathrm{em}$ relação ao SR (1.92 $\pm 0.38 \mathrm{mmol} . \mathrm{L}-1)$. Apesar de intensidades diferentes do LAn entre os exercícios, as variáveis FC e PSE não apresentaram diferenças significativas. Foi possível identificar o LAn em idosos nos aparelhos LP e SR, ocorrendo em intensidades inferiores no SR.

Palavras-chave: treinamento de força, limiar anaeróbio, lactacidemia, exercício incremental, terceira idade
\end{abstract}

ABSTRACT

The presence of anaerobic threshold (AT) in resistance exercise has been studied in recent years. The aim of this study was to identify and to compare the AT in elderly population during leg press (LP) and bench press $(\mathrm{BP})$. Fourteen elderly men $(68.9 \pm 4.0$ years, $170.5 \pm 0.05 \mathrm{~cm}$ e $76.1 \pm 7.8 \mathrm{~kg})$ performed two maximal incremental tests based on percentages of one repetition maximum (1RM) for the determination of AT during LP and BP. Blood lactate, heart rate (HR) and rating of perceived exertion (RPE) were measured at the final of each stage and it were compared between the exercises at the intensity of the AT. The level of significance was $p \leq .05$. The AT in the LP was $27.9 \pm 5.0 \%$ of $1 \mathrm{RM}$ and in the $\mathrm{BP}$ was $21.5 \pm 3.1 \%$ of $1 \mathrm{RM}$, with statistical differences between the exercises. However, blood lactate was significantly lower on LP $(1.36 \pm 0.40 \mathrm{mmol}$.L-1) when compared with BP (1.92 $\pm 0.38 \mathrm{mmol} . \mathrm{L}-1)$. Although the intensity of AT has been different between the exercises, HR and RPE did not presented statistical significant differences. It was possible to identify the AT on elderly population during LP and BP exercises and it occurred in lower intensities for BP. Keywords: resistance training, anaerobic threshold, blood lactate, incremental exercise, older

Submetido: 05.03.2012 | Aceite: 17.08.2012

Nuno Manuel Frade de Sousa, Vilmar Baldissera. USP, São Carlos/SP, Brasil.

Markus Vinicius Campos Souza, Guilherme Borges Pereira, Danilo Rodrigues Bertucci, Rodrigo Ferro Magosso, Sérgio Eduardo Perez de Andrade. Universidade Federal de São Carlos, São Carlos/SP, Brasil.

Endereço para correspondência: Markus Vinicius Campos Souza, Laboratório de Fisiologia do Exercício, Departamento de Ciências Fisiológicas, UFSCar, Rod Washington Luis, km 235 Cp 676, CEP 13565 - 905

São Carlos (SP), Brasil.

E-mail: markuscampos@hotmail.com 
O envelhecimento está associado a mudanças fisiológicas e ao declínio das capacidades físicas, induzindo a diminuição na força e funcionalidade do idoso (Chodzko-Zajko, et al., 2009). É recomendável, então, meios que melhorem a qualidade de vida e os tornem menos vulneráveis a diferentes tipos de distúrbios físicos e fisiológicos. O exercício resistido tem sido enfaticamente incentivado para pessoas idosas, a fim de aprimorar as capacidades físicas e reserva funcional. Este tipo de exercício visa preservar e desenvolver as funções cardiovasculares, ventilatórias e metabólicas em um nível mais elevado que os indivíduos sedentários de idade equivalente (Chodzko-Zajko, et al., 2009; Kraemer, et al., 2002; Stratton, Levy, Cerqueira, Schwartz, \& Abrass, 1994).

Recentemente, em estudos realizados com adultos e idosos saudáveis, foi observado o ponto de transição metabólica entre o aeróbio e anaeróbio durante exercício resistido incremental (Barros, Agostini, Garcia, \& Baldissera, 2004; Moreira, et al., 2008; Simoes, et al., 2010). Este ponto ocorre geralmente em intensidades moderadas e com um elevado número de repetições, intensidades estas mais efetivas para melhorar a resistência muscular localizada e aptidão cardiorrespiratória em indivíduos saudáveis idosos (ACSM, 2009).

O primeiro a estudar o comportamento da curva de lactacidemia em exercício resistido foi Barros et al. (2004), que determinou o limiar anaeróbio (LAn) em indivíduos jovens treinados e destreinados nos exercícios leg press (LP) e rosca direta. O LAn também já foi determinado em uma população de idosos através de dois métodos diferentes, lactacidemia e variabilidade da frequência cardíaca (Simoes, et al., 2010). No entanto, o estudo foi realizado apenas no aparelho LP. Apesar de faixas etárias e aparelhos diferentes, todas pesquisas encontraram a intensidade do LAn próximo de $30 \%$ de 1RM (Barros, et al., 2004; Moreira, et al., 2008; Simoes, et al., 2010).

A possibilidade de treinamento resistido na intensidade do LAn em uma população idosa pode orientar os treinadores no planejamento do nível de intensidade que é necessário para melhorar as capacidades musculares que são estimuladas pelo treinamento resistido nesta população. Além disso, este tipo de treinamento, com elevado número de repetições e intensidade moderada, poderá ser suficiente para gerar benefícios na população idosa, além de diminuir riscos (Glass \& Stanton, 2004). Entretanto, devido ao escasso número de evidências do LAn em exercício resistido em idosos, faz-se necessário um estudo mais aprofundado de todas as suas variáveis. Principalmente se a intensidade do LAn é semelhante em vários exercícios resistidos, facilitando a prescrição do exercício, ou se difere entre exercícios.

Assim, os objetivos deste trabalho foram identificar e comparar o LAn em uma população idosa nos exercícios LP e supino reto (SR) e comparar as variáveis frequência cardíaca (FC), lactacidemia e percepção subjetiva de esforço (PSE) na intensidade do LAn para o SR e LP. Hipotetizamos que é possível determinar o LAn em intensidades semelhantes em ambos os exercícios.

\section{MÉTODO}

\section{Amostra}

Participaram do estudo 14 homens idosos com idade de $68.9 \pm 4.0$ anos, estatura média de $170.5 \pm 0.05 \mathrm{~cm}$ e peso médio de $76.1 \pm$ $7.8 \mathrm{~kg}$. Todos os voluntários tinham experiência prévia de pelo menos seis meses em exercícios resistidos de hipertrofia com cargas típicas correspondendo 6 a 12 RM.

O experimento foi aprovado pelo Comitê de Ética local em pesquisa. Os participantes foram informados detalhadamente sobre os procedimentos utilizados e concordaram em participar voluntariamente do estudo, assinando o termo de consentimento informado e proteção da privacidade. 


\section{Desenho experimental}

O estudo foi desenvolvido em dois aparelhos distintos, o LP e o SR. Estes aparelhos de musculação foram escolhidos devido à sua elevada popularidade nas academias de musculação, além de poderem ser comparadas respostas entre membros inferiores e superiores. Para cada aparelho foram realizadas três sessões de testes, com intervalo entre as sessões de 48 horas. Os testes no SR foram aplicados na semana seguinte ao LP.

$\mathrm{Na}$ primeira sessão foi determinada uma repetição máxima (1RM), sendo que na segunda foi realizado o re-teste a fim de minimizar os erros nos valores obtidos de $1 \mathrm{RM}$. Na terceira sessão, o teste incremental máximo baseado em porcentagens de 1RM identificou o LAn.

Os voluntários foram orientados a não praticar nenhum exercício exaustivo nos dias anteriores aos testes, a fim de não alterar as respostas fisiológicas dos mesmos.

\section{Teste de uma repetição máxima (1RM)}

Antes do teste, os participantes realizaram uma breve adaptação em cada aparelho, de modo a estabelecer a correta biomecânica do exercício. Foram instruídos a executar os movimentos da seguinte forma: (i) cada repetição com duração de três segundos, sendo 1.5 segundos para a fase concêntrica e 1.5 segundos para a fase excêntrica, controlado através de comandos visuais e verbais; (ii) a padronização das angulações de movimentos seguiram as descrições de Brown e Weir (2001), controladas por ângulo de movimento da articulação do joelho e do cotovelo entre 90 e 180 graus um eletrogoniômetro (EMG System do Brasil, São José dos Campos, SP, Brasil).

Após a breve adaptação, os voluntários realizaram o teste de 1RM, como sugerido por Kramer e Fry (1995). O aquecimento consistiu na execução de oito repetições com $50 \%$ de 1RM estimada (baseada em testes pilotos e percepção da força máxima individual). Após dois minutos de repouso foram realizadas mais três repetições a 70\% de 1RM estimada. Os estágios seguintes consistiam na determinação de 1RM, com intervalos de cinco minutos entre cada tentativa. O re-teste foi realizado 48 horas após a determinação de 1RM. Neste caso, após aquecimento, como descrito anteriormente, o voluntário iniciava o teste com a carga estabelecida na sessão anterior. O valor máximo obtido entre os dois testes foi assumido como 1RM, sendo que a diferença não poderia ser maior do que $5 \%$ entre eles.

\section{Teste incremental}

O teste incremental foi realizado 48 horas após a segunda sessão de determinação de 1RM. A seleção das intensidades para o teste foram 10, 20, 25, 30, 35, 40, 50, 60 e $70 \%$ de 1RM. Esta divisão de intensidades foi escolhida devido aos resultados de estudos prévios, que determinaram o LAn próximo a 30\% de 1RM (Barros, et al., 2004; Moreira, et al., 2008; Simoes, et al., 2010).

Em cada intensidade foram executadas 20 repetições com duração de um minuto (três segundos por repetição). Entre cada série houve um repouso passivo, que serviu para ajuste da próxima carga e coleta de sangue para determinação da concentração de lactato. $\mathrm{O}$ teste foi dado como encerrado quando o voluntário não conseguia realizar as repetições dentro da biomecânica estabelecida ou quando não completava 20 repetições para cada intensidade.

\section{Concentração de lactato sanguíneo}

Trinta segundos após o término de cada estágio foram coletados $25 \mu \mathrm{l}$ de sangue capilarizado do lóbulo da orelha, através de capilares heparinizados previamente calibrados (Goodwin, Harris, Hernandez, \& Gladden, 2007). Imediatamente após a coleta, a concentração de lactato sanguíneo foi determinada através do método eletro-enzimático (1500 Sport, Yellow Springs Instruments Inc., USA). 


\section{Frequência Cardíaca}

A FC foi continuamente mensurada através de um monitor cardíaco (Polar S610, Kempele, Filândia). Para a sua análise, foi registrada em repouso (um minuto antes do início do teste) e na $18^{\mathrm{a}}$ repetição de cada intensidade. A equação de Tanaka (Tanaka, Monahan, \& Seals, 2001) foi utilizada para a predição da FC máxima $\left(\mathrm{FC}_{\max }\right)$.

\section{Percepção Subjetiva de Esforço}

Foram utilizadas duas escalas para a análise da PSE - escala de BORG (Borg, 1978) e de OMNI (Robertson, et al., 2003). A escala de Borg foi utilizada para relatar o esforço geral percebido no corpo e a de OMNI apenas a do grupamento muscular recrutado no movimento. Cada voluntário indicava a PSE imediatamente após o final de cada intensidade.

\section{Determinação do LAn}

A determinação do Lan foi realizada através de um modelo de ajuste algorítmico com a utilização de uma regressão linear dupla (Sousa, et al., 2011). Resumidamente, foi realizada uma análise de regressão linear computorizada, dividindo o conjunto de pontos da concentração de lactato sanguíneo em dois segmentos de reta. $\mathrm{O}$ ponto de intercepção entre os dois segmentos foi considerado a intensidade do Lan. O Matlab versão 7.4 (MathWorks, USA) foi utilizado para o cálculo.

\section{Análise estatística}

Os resultados são expressos em média e desvio padrão. Todas as variáveis analisadas apresentaram distribuição normal (Shapiro-Wilk). O teste $\mathrm{t}$ de student para amostras independentes foi utilizado para comparar as diferentes variáveis entre o LP e SR. O software utilizado foi o SPSS versão 17.0 (Somers, NY, USA) com nível de significância aceite de $p \leq .05$.

\section{RESULTADOS}

A média \pm SD de 1 RM para o aparelho LP foi $238.2 \pm 53.4 \mathrm{~kg}$ e para o SR foi $58.1 \pm 6.3$. $\mathrm{O}$ coeficiente de correlação entre o primeiro teste e o re-teste foi 0.99 para o LP e 0.96 para o SR.

A tabela 1 apresenta os principais resultados na intensidade máxima atingida durante o teste incremental. A intensidade máxima atingida no LP foi estatisticamente superior ( $p=$ .02) ao aparelho SR. A concentração máxima de lactato sanguíneo também foi estatisticamente superior no LP em relação ao SR ( $p=$ .01). Não foram observadas diferenças estatisticamente significativas entre os aparelhos nos parâmetros FC $(p=.52)$, esforço percebido na escala de BORG $(p=.22)$ e na escala de OMNI $(p=.31)$ na intensidade máxima do teste incremental.

Tabela 1

Valores de concentração de lactato sanguíneo, frequência cardíaca (FC), percentual da frequência cardíaca máxima (\%FCmax) e percepção subjetiva de esforço na intensidade máxima (\%1RM) atingida no teste incremental.

\begin{tabular}{ccc}
\hline & \multicolumn{2}{c}{ Intensidade Máxima } \\
\cline { 2 - 3 } & LP & SR \\
\hline \%1RM & $64.5(6.9)^{*}$ & $56.7(7.1)$ \\
Lactato (mmol.L-1) & $5.85(0.83)^{*}$ & $4.38(1.18)$ \\
FC (bpm) & $119.7(3.6)$ & $124.3(7.6)$ \\
\%FCmax & $75.1(8.9)$ & $77.9(11.2)$ \\
BORG & $19.2(1.4)$ & $18.0(2.6)$ \\
OMNI & $9.3(1.10)$ & $8.4(2.4)$ \\
\hline
\end{tabular}

${ }^{*}$ Diferença estatisticamente significativa $(p \leq .05)$ entre leg press (LP) e supino reto (SR).

A tabela 2 apresenta os principais resultados do teste incremental na intensidade relativa ao LAn. Foi possível determinar o LAn para todos os voluntários por meio do modelo de ajuste algorítmico. A intensidade do LAn foi estatisticamente superior no LP em relação ao SR $(p<01)$. No entanto, a concentração de lactato sanguíneo na intensidade do LAn foi estatisticamente inferior no LP $(p<.01)$. A figura 1 mostra a intensidade máxima e do LAn para cada exercício executado.

Assim como no final do teste incremental, a 
FC $(p=.24)$, o esforço percebido na escala de BORG $(p=.57)$ e na escala de OMNI $(p=.37)$ não apresentaram diferenças estatisticamente significativas entre os aparelhos na intensidade do LAn.

\section{Tabela 2}

Valores de concentração de lactato sanguíneo, frequência cardíaca $(F C)$, percentual da frequência cardíaca máxima (\%FCmax) e percepção subjetiva de esforço na intensidade do limiar anaeróbio (\%1RM).

\begin{tabular}{ccc}
\hline & \multicolumn{2}{c}{ Intensidade do limiar anaeróbio } \\
\cline { 2 - 3 } & LP & SR \\
\hline \%1RM & $27.9(5.0)^{*}$ & $21.5(3.1)$ \\
Lactato (mmol.L-1) & $1.36(0.40)^{*}$ & $1.92(0.38)$ \\
FC (bpm) & $97.1(9.2)$ & $92.2(8.4)$ \\
\%FCmax & $60.9(5.7)$ & $57.8(5.3)$ \\
BORG & $10.3(3.1)$ & $9.6(2.4)$ \\
OMNI & $3.3(2.5)$ & $2.4(1.2)$ \\
\hline
\end{tabular}

*Diferença estatisticamente significativa $(p \leq .05)$ entre leg press (LP) e supino reto (SR).

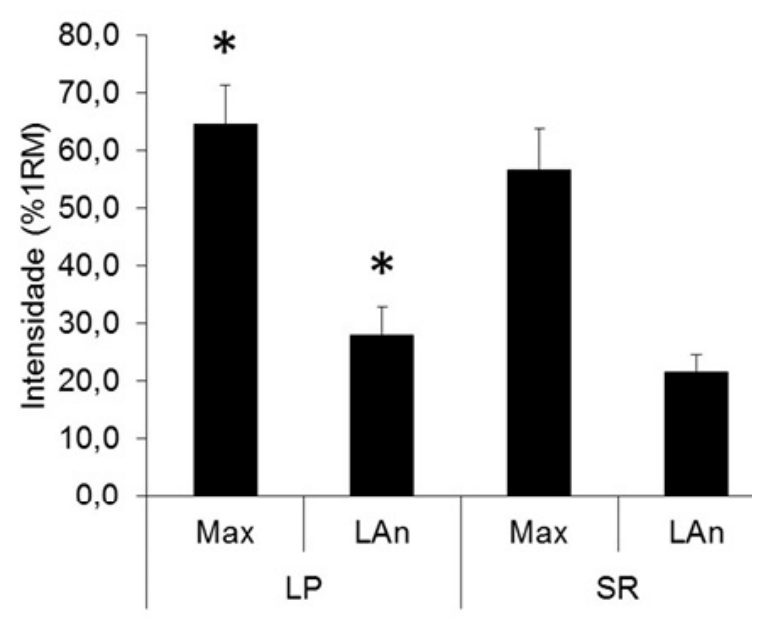

Figura 1 Intensidade máxima (Max) e do limiar anaeróbio (LAn) nos aparelhos leg press (LP) e supino reto (SR). *Diferença estatisticamente significativa entre LP e SR $(p<.05)$

\section{DISCUSSÃO}

O presente estudo evidenciou que é possível determinar o LAn por meio de um exercício crescente no SR e LP em uma população idosa, sendo esses valores de $21.5 \%$ e $27.9 \%$ de $1 \mathrm{RM}$, respectivamente. A intensidade do LAn, assim como a concentração de lactato, neste ponto foram diferentes entre os dois exercícios estudados. Por outro lado, os valores de FC e PSE atingidoos nessa intensidade são semelhantes.

Durante a realização dos exercícios, a intensidade alcançada no LP foi maior que no SR. Esses resultados são esperados, uma vez que o grupamento muscular proveniente dos membros inferiores utilizado para a realização do exercício LP é maior que no SR, permitindo a realização de um maior número de repetições e retardando a fadiga através da participação de um maior número de músculos (Shimano, et al., 2006). Além disso, para uma mesma intensidade expressa em percentual de 1RM, indivíduos realizam um maior número de repetições para membros inferiores quando comparado a membros superiores, independente da velocidade do movimento (Hatfield, et al., 2006; Willardson \& Burkett, 2006). Como consequência desta máxima intensidade entre os exercícios, a lactacidemia também foi significativamente superior no LP em comparação ao SR. Assim, o maior grupamento muscular recrutado no exercício LP juntamente com a maior intensidade alcançada podem justificar o término do exercício LP com maiores concentrações de lactato sanguíneo, quando comparado com o SR.

Apesar da exaustão ocorrer em intensidades diferentes no LP e SR, os valores correspondentes de PSE e FC foram semelhantes. Como os exercícios foram realizados até a exaustão, os resultados encontrados na pesquisa referentes à PSE estão de acordo com a literatura, uma vez que são esperadas PSE próximas ao máximo (Lagally, et al., 2002; Tiggemann, 2010). Por outro lado, a FC não atingiu valores máximos, contrariamente a testes aeróbios dinâmicos que são frequentemente utilizados para determinar a frequência cardíaca máxima (Foster, et al., 2007). No entanto, os valores de pico da FC foram semelhantes ao estudo de Ratamess et al. (2007), que também analisou a resposta da FC durante o exercício resistido. A natureza 
dos exercícios não permite o aumento da FC até aos seus valores máximos previstos, mostrando que uma série de 20 repetições com intensidades entre 50 a $70 \%$ de $1 \mathrm{RM}$ é insuficiente para atingir a FC máxima prevista, mesmo quando o exercício é realizado até à exaustão.

O principal objetivo do estudo foi alcançado, já que foi possível determinar o LAn em idosos durante o exercício resistido, assim como outras pesquisas que haviam determinado em diferentes populações (Barros, et al., 2004; Moreira, et al., 2008; Simoes, et al., 2010). Porém, este estudo apresenta resultados contraditórios à pesquisa de Barros et al (2004), uma vez que o LAn ocorreu em intensidades diferentes para os dois exercícios.

A maioria dos estudos envolvendo LAn em exercício resistido indica como a principal causa para esse fenômeno o fator hemodinâmico (Barros, et al., 2004; Moreira, et al., 2008). Esse fator está relacionado ao colabamento dos capilares, ocasionada pela pressão intramuscular aumentada, principalmente na fase concêntrica, diminuindo o fluxo sanguíneo (Petrofsky, Phillips, Sawka, Hanpeter, \& Stafford, 1981). Outros fatores também já foram descritos na tentativa de explicar o LAn em exercícios resistidos, entre eles a estimulação das fibras musculares e a ativação simpática (Simoes, et al., 2010). Devido à diminuição no fluxo sanguíneo, ocorre a estimulação das fibras glicolíticas de contração rápida no músculo esquelético, promovendo mudanças metabólicas na musculatura, como a redução do pH. Essas informações são enviadas para a região ventrolateral bulbar, desencadeando um aumento na descarga simpática no sistema cardiovascular, podendo esta atividade aumentar a secreção de catecolaminas pela glândula adrenal.

Esse conjunto de fatores pode ajudar a justificar as diferenças na intensidade do LAn entre os dois exercícios encontrados neste estudo. Hargreaves et al. (1988) verificaram que para uma mesma intensidade relativa de esforço, ocorre maior liberação de lactato nos movi- mentos realizados com os braços em comparação com as pernas, devido à maior glicogenólise muscular nos membros superiores, ocasionada por maior predominância de fibras glicolíticas. Desse modo, o maior trabalho realizado pelas fibras glicolíticas nos membros superiores poderá representar uma maior liberação de lactato em intensidades de exercício semelhantes.

O aumento da FC cardíaca foi bastante discreto até à intensidade do LAn, podendo ser um indicador de segurança cardiovascular na realização deste tipo de exercício para idosos. Além disso, os baixos valores de PSE se devem às baixas cargas, o que faz com que o idoso sinta mais segurança na execução do exercício. Devido à semelhança entre estes dados nos dois aparelhos, é importante realizar novos estudos a fim de verificar se este comportamento é padrão para os exercícios resistidos. A confirmação destes resultados poderão permitir a identificação do LAn por um método indireto.

Existem algumas limitações do estudo que devem ser consideradas, nomeadamente o estado e tipo de treinamento da população idosa, duas variáveis do treinamento resistido que podem influenciar diretamente os resultados obtidos. O LAn pode apresentar intensidades diferentes dependendo do tipo de treinamento realizado. O grupo estudado tinha experiência prévia em treinamento de hipertrofia, entretanto, estudos recentes indicam que o treinamento de potência é o mais indicado para este tipo de população, o que poderia alterar os resultados. Além disso, são necessários estudos com idosos sedentários, tipo de população que também pode ser beneficiada com este tipo de treinamento. Considerando as devidas limitações da pesquisa, foi possível verificar que o LAn pode ser diferente entre aparelhos, apesar da diferença ser menor que $10 \%$ de 1RM. Desta forma, a possibilidade de prescrição de treinamento resistido através do LAn deve considerar diferentes intensidades para diferentes exercícios de musculação. Sem 
dúvida que novos estudos relativos a esta temática devem ser desenvolvidos, pesquisando diferentes exercícios de musculação e principalmente o efeito de um programa de treinamento resistido baseado na intensidade do LAn. Pesquisas relativas ao efeito de um programa de treinamento são essenciais para determinar a importância do LAn em exercício resistido. Todavia, a utilização de intensidades leves a moderadas são recomendadas pelo American College of Sports Medicine para o desenvolvimento de força e potência muscular em idosos (Garber, et al., 2011). Este tipo de treinamento pode minimizar os riscos de lesões musculares por sobrecarga aos sistemas cardiovascular e muscular (ACSM, 2009).

\section{CONCLUSÕES}

Concluindo, apesar das respostas de FC e PSE serem semelhantes entre os exercícios LP e SR, o limiar anaeróbio ocorreu em intensidades inferiores no aparelho SR. A concentração de lactato sanguíneo na intensidade do limiar anaeróbio também foi superior no aparelho SR. Além disso, a intensidade máxima atingida durante o teste crescente foi superior no aparelho LP, assim como a respetiva concentração de lactato sanguíneo.

\section{Agradecimentos:}

$O$ autor principal foi financiado pela Fundação para a Ciência e Tecnologia (FCT) - Ministério da Educação e Ciência, Portugal (MCTES; Ref. SRFH/ $\mathrm{BD} / 46898 / 2008)$.

\section{Conflito de Interesses:}

Nada declarado.

\section{Financiamento:}

Nada declarado.

\section{REFERÊNCIAS}

ACSM (2009). American College of Sports Medicine position stand. Progression models in resistance training for healthy adults. Medicine and
Science in Sports and Exercise, 41 (3), 687-708.

Barros, C. L., Agostini, G. G., Garcia, E. S., \& Baldissera, V. (2004). Limiar de Lactato em Exercício Resistido. Revista Motriz, 10(1), 31 - 36.

Borg, G. (1978). Subjective effort and physical abilities. Scandinavian Journal of Rehabilitation Medicine Supplement, 6, 105-113.

Brown, L. E., \& Weir, J. (2001). Procedures Recommendation I: Accurate Assessment Of Muscular Strength And Power. Journal of Exercise Physiology online, 4(3), 1 - 21.

Chodzko-Zajko, W. J., Proctor, D. N., Fiatarone Singh, M. A., Minson, C. T., Nigg, C. R., Salem, G. J., et al. (2009). American College of Sports Medicine position stand. Exercise and physical activity for older adults. Medicine and Science in Sports and Exercise, 41 (7), 1510-1530.

Foster, C., Kuffel, E., Bradley, N., Battista, R. A., Wright, G., Porcari, J. P., et al. (2007). VO2max during successive maximal efforts. European Journal of Applied Physiology, 102 (1), 67-72.

Glass, S. C., \& Stanton, D. R. (2004). Self-selected resistance training intensity in novice weightlifters. Journal of Strength and Conditioning Research, 18(2), 324-327.

Goodwin, M. L., Harris, J. E., Hernandez, A., \& Gladden, L. B. (2007). Blood lactate measurements and analysis during exercise: a guide for clinicians. Journal of Diabetes Science and Technology, 1 (4), 558-569.

Hargreaves, M., \& Richter, E. A. (1988). Regulation of skeletal muscle glycogenolysis during exercise. Canadian Journal of Sport Sciences, 13(4), 197-203.

Hatfield, D. L., Kraemer, W. J., Spiering, B. A., Hakkinen, K., Volek, J. S., Shimano, T., et al. (2006). The impact of velocity of movement on performance factors in resistance exercise. Journal of Strength and Conditioning Research, 20(4), 760-766.

Kraemer, W. J., Adams, K., Cafarelli, E., Dudley, G. A., Dooly, C., Feigenbaum, M. S., et al. (2002). American College of Sports Medicine position stand. Progression models in resistance training for healthy adults. Medicine and Science in Sports 
and Exercise, 34(2), 364-380.

Kraemer, W. J., \& Fry, A. C. (1995). Strength testing: development and evaluation of methodology. Champaign, I.L.: Human Kinetics.

Moreira, S. R., Arsa, G., Oliveira, H. B., Lima, L. C., Campbell, C. S., \& Simoes, H. G. (2008). Methods to identify the lactate and glucose thresholds during resistance exercise for individuals with type 2 diabetes. Journal of Strength and Conditioning Research, 22(4), 1108-1115.

Petrofsky, J. S., Phillips, C. A., Sawka, M. N., Hanpeter, D., \& Stafford, D. (1981). Blood flow and metabolism during isometric contractions in cat skeletal muscle. Journal of Applied Physio$\log y, 50(3)$, 493-502.

Ratamess, N. A., Falvo, M. J., Mangine, G. T., Hoffman, J. R., Faigenbaum, A. D., \& Kang, J. (2007). The effect of rest interval length on metabolic responses to the bench press exercise. European Journal of Applied Physiology, $100(1), 1-17$.

Robertson, R. J., Goss, F. L., Rutkowski, J., Lenz, B., Dixon, C., Timmer, J., et al. (2003). Concurrent validation of the OMNI perceived exertion scale for resistance exercise. Medicine and Science in Sports and Exercise, 35(2), 333-341.

Simoes, R. P., Mendes, R. G., Castello, V., Machado, H. G., Almeida, L. B., Baldissera, V., et al. (2010). Heart-rate variability and blood-lactate threshold interaction during progressive resistance exercise in healthy older men. Journal of Strength and Conditioning Research, 24(5), 13131320.
Sousa, N. M. F. d., Magosso, R. F., Pereira, G. B., Leite, R. D., Arakelian, V. M., Montagnolli, A. N., et al. (2011). The measurement of lactate threshold in resistance exercise: a comparison of methods. Clinical Physiology and Nuclear Medicine.

Stratton, J. R., Levy, W. C., Cerqueira, M. D., Schwartz, R. S., \& Abrass, I. B. (1994). Cardiovascular responses to exercise. Effects of aging and exercise training in healthy men. Circulation, 89(4), 1648-1655.

Tanaka, H., Monahan, K. D., \& Seals, D. R. (2001). Age-predicted maximal heart rate revisited. Journal of American College of Cardiology, 37(1), 153-156.

Tiggemann, C. (2010). A PERCEPÇÃO DE ESFORÇO NO TREINAMENTO DE FORÇA. Revista Brasileira de Medicina do Esporte, 16(4).

Willardson, J. M., \& Burkett, L. N. (2006). The effect of rest interval length on the sustainability of squat and bench press repetitions. Journal of Strength and Conditioning Research, 20(2), 400-403.

(cc) EY-NC Todo o conteúdo da revista Motricidade está licenciado sob a Creative Commons, exceto quando especificado em contrário e nos conteúdos retirados de outras fontes bibliográficas. 